\title{
Safety Strategies in Hospitals
}

We have developed a series of ideas and proposals in the book which together laid the foundations for five safety strategies described in Chap. 6. We believe that thinking of safety strategies in this way has three major advantages: first, we can enlarge the range of safety strategies and interventions available to us; secondly we can customise the blend of strategies to different contexts and third the high level architecture of safety strategies may help us think more strategically about safety both day to day and on a long term basis

In this chapter we begin the process of exploring how these strategies might support safety in the hospital. The following chapters address home care and primary care. In each case we provide a short introduction to relevant aspects of safety in each context but do not dwell on well-established findings. Our primary purpose is to provide examples of interventions associated with each of the five strategies and to give a sense of the potential value of such an approach. We recognise that, in the longer term, considerable further empirical work would be needed to develop and confirm (or discount) our proposals.

\section{A Little History}

Hospital care has been the main focus of patient safety for two decades now and we can distinguish a series of phases of exploration and intervention. Each phase brought some success but simultaneously revealed barriers and limitations, which in turn stimulated a new phase of work in an evolving trial and error strategy. With experience and maturity, we understand more today about what is achievable and what has proved illusory. We are much more aware of how difficult it is to improve safety in both the short and long term.

What has been done in past decades? In the past 15 years we can distinguish three main phases each associated with different types of action and intervention. The earlier strategies have continued as the new ones emerged so that we now have 'a safety layer cake' of practices and interventions. 


\section{The Enthusiasm of the Early Years, 1995-2002}

Systematic work on patient safety began in the mid 1990s with an emerging demarcation between a broad concern with quality and a specific focus on harm. In Britain for instance the development of clinical risk management, initially targeted at the reduction of litigation, brought a new emphasis on the analysis and reduction of harmful incidents and events (Vincent 1995). The methods and assumptions however remained rooted in those of quality improvement; the aim was to identify and count errors and incidents and then find ways of preventing them. Establishing reporting systems to detect and record incidents was at the core of the strategy. This approach was rapidly reconsidered as a result of both massive under-reporting, especially from doctors, and a gradual realisation of the impossibility of resolving the growing number of problems identified in reporting systems (Stanhope et al. 1999). A wider vision was needed which was provided by systemic concepts and tools imported from industry.

\section{The Advent of Professionalism 2002-2005}

In the late 1990s, James Reason provided an inspirational vision for healthcare that provided a clear demarcation between traditional approaches to quality improvement and the specific problems that arise when addressing safety (Reason 1997; Reason et al. 2001). Safety researchers, clinicians and managers took the concepts, techniques and methods from industrial safety and applied them to healthcare. These included a stronger emphasis on the role of latent organisational conditions which led to the development of methods of incident analysis derived this model (Vincent et al. 1998, 2000). Increasing attention was also given to human factors and ergonomics, following the success in improving interface and equipment design in industry, the use of information technology and a scientific approach to working conditions, stress and fatigue management (Bates 2000; Sexton et al. 2000; Carayon 2006). Accreditation and certification built on this new knowledge in requiring hospitals to establish risk management programmes and new patient safety indicators. Safety and risk management acquired a much higher profile and many new initiatives were developed across the healthcare system, but the impact on the safety of patients remained uncertain (Pronovost et al. 2006; Wachter 2010). The lack of clinical engagement was a major concern with patient safety remaining the province of enthusiasts and specialists - a curious situation given that safety, considered in terms of personal accountability, is perhaps the dominant concern of clinicians in their day-to-day work with patients.

\section{Safety Culture, Multifaceted Interventions, and Teamwork 2005-2011}

Surveys of safety culture demonstrated unequivocally that in many hospitals and other healthcare settings safety attitudes and values were far from ideal. Findings from many studies suggested an excessive blame culture, pressure on performance 
to the detriment of safety, little transparency towards patients and variable levels of supervision and teamwork. There was also huge variability between hospitals, within clinical disciplines and across different settings (Tsai et al. 2013). Whereas safety culture was initially seen as potentially directly impacting on safety, there was now a growing awareness that it might provide only a necessary foundation (Flin et al. 2006; Vincent et al. 2010)

However, as we have discussed, evidence began to emerge of marked improvements in specific safety problems at a local level and of the potential of wider application of approaches such as checklists, care bundles and so on (Haynes et al. 2009; Shekelle et al. 2011). Those proven safety wins on the frontline encouraged the healthcare community to believe that safety would progressively improve as more interventions were put into place. Improving safety across organisations and populations however has proved a great deal more challenging. The major difference between current views and what was imagined in the mid 2000s is that safety wins and rewards are now expected in the middle to long term rather than in the very short term.

\section{Reflections on Safety in Hospitals}

We provide this brief overview primarily to highlight the fact that approaches to safety in hospitals have primarily been optimising approaches of one kind and another, although comparatively little attention has been given to optimising the system overall as opposed to improving specific practices. Accreditation and regulations of the system might be thought to be examples of risk control and there are certainly examples of standards being set in order to minimise or avoid risks of certain kinds. However we suggest that most accreditation and regulation is essentially aimed at assessing compliance or failure to comply with defined standards of care. Regulators are sometimes forced to acknowledge that standards cannot be met and that adaptations must be made but we suggest that the dominant vision of how safety is achieved is one of adherence to standards.

\section{Safety in Hospital: Distinguishing Current and Future Strategies}

We propose that thinking in terms of an overall blend of high level safety strategies customised to different contexts will be an efficient and effective approach both to managing safety on a day to day basis and to improving safety over the long term. However before we start to illustrate how the five different strategies might be employed in hospital we need to consider a critical issue, which is that staff and organisations often have to employ a particular strategy not because of the needs of that clinical environment but to compensate for other underlying problems in the system. For instance, services such as acute medicine rely very heavily on monitoring, adaptation and recovery to observe, correct and recover from the inevitable departures from best practice and unforeseen problems that arise. However the fact 
that a strategy is extensively used does not necessarily mean that it is desirable; it might in fact be overused to compensate for other deficiencies such as poor reliability or inadequate staffing (Box 7.1). We therefore need to make a distinction at this point between:

- The blend of strategies currently used by an organisation

- The blend that might be desirable

- The strategies that might need to be developed or enhanced

\section{Box 7.1. Adaptation and Compromise on the Wards}

Recently while on call at the weekend I found my team looking dispirited, ploughing through 27 pages of printed jobs that were required for patients based on ten wards. There was no way these could all be done by two junior doctors. They were doing what any sensible person would do and "working round" an impossible task, rationing what was essential or urgent and what could be omitted.

A large proportion of the workload is phlebotomy, taking bloods and chasing the results. These should be taken by technicians but they have a fixed contract for $4 \mathrm{~h}$ meaning that they only deal with a small proportion of the overall workload. Tests are ordered by weekday teams, and handed over to the weekend team to check results, often without a clear indication of the purpose of the tests or what to do with the results. The weekend teams only become aware when blood has not been taken when they check for the result, leading to considerable delay in monitoring patients. There is huge variability in the clarity of the requests, the background information given, the appropriateness of the job itself and what to do with results, all compounded by the inexperience and insecurity of junior doctors on call at weekends.

Inada Kim (personal communication 2015)

Staff in all environments rely on workarounds such as obtaining information from patients rather than their health records, or using disposable gloves as tourniquets. In some cases, risks are taken such as making clinical decisions without information, or transferring used sharps to sharps bins in remote locations (Burnett et al. 2011). Often front-line coping and adaptation leads to short-term "fixes" that put off more fundamental, long-term solutions. These clinical work-arounds may also allow managers to protect themselves from inconvenient truths and shift accountability for failure to front-line workers (Wears and Vincent 2013).

We therefore always need to think, when formulating the overall approach to safety, both about what the approach is now and what might be the most effective strategy in the longer term. We certainly believe that adaptive strategies should be further developed in the sense of being planned and to some extent formalised. However this is very different from the current reliance on ad hoc improvising to compensate for missing information, faulty equipment and the like. Figure 7.1 


\section{Improving systems reduces the need \\ for adaptation and recovery}

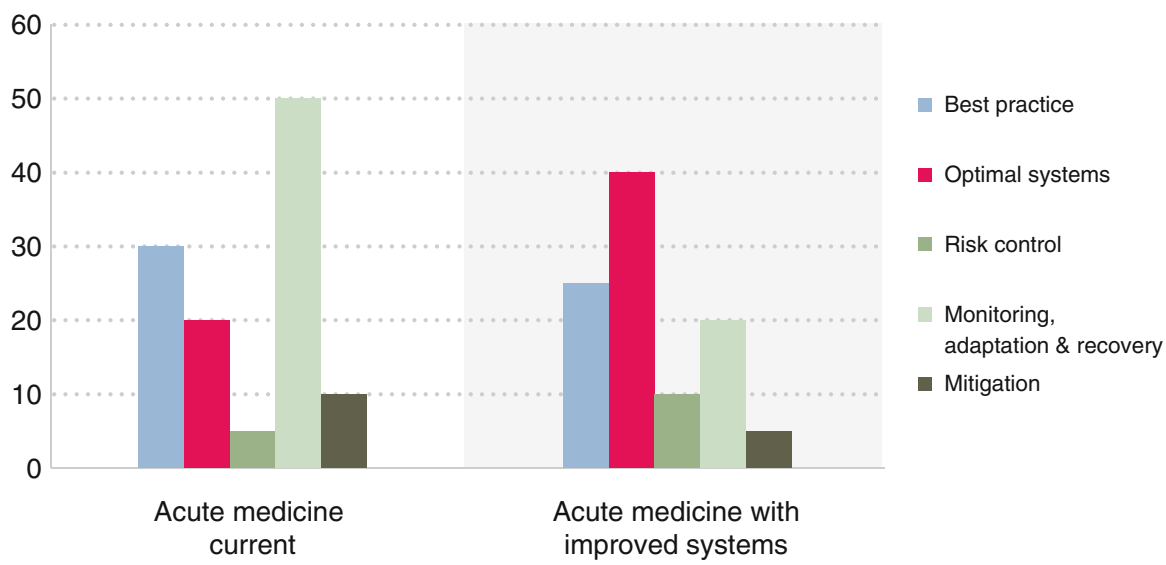

Fig. 7.1 Improving systems reduces the need for adaptation

illustrates these ideas in the context of acute medicine suggesting that increasing reliability and controlling flow and demand would reduce the need for adaptation and improvisation. With this in mind we now illustrate the five safety strategies in the context of the hospital; we devote most space to risk control, adaptation and mitigation as the other two strategies are already well described.

\section{Safety as Best Practice}

We have already given a number of examples of optimising strategies in hospitals in Chap. 6 and earlier in the book. Clearly one needs to consolidate and develop the approaches that aim to improve adherence to best clinical practice and thereby make care safer for patients. Reduction of pressure ulcers, reduction of catheter-associated infections, improved hand hygiene, improved patient identification and so on are obviously critical. Such standardised tasks and processes can be routinely audited to ensure that standards are maintained. All hospital environments, no matter how fluid and dynamic the workflow, have many core basic procedures which need to be followed. Programmes to improve adherence to basis procedures are always an important foundation for safety though never a complete solution.

\section{Improving the System}

Although the broad field of human factors and ergonomics is both huge and critically important in hospital settings we will not consider it in detail here. This is because it has been extensively discussed elsewhere and accepted as a valid and 
essential approach to improving safety as well as effectiveness and experience. Under this broad heading we would include improvements to the administration of medication in terms of standardizing formularies and protocols, the introduction of information technology in all its forms, formalising roles and responsibilities in clinical teams, the use of care bundles and daily goals to organise ward care and all efforts to improve basic working conditions. Improving safety through best practice and raising standards tends to require additional effort from frontline staff, at least in the early stages. There is an equal need to give attention to improving the system in order to reduce the burden on staff and so allowing more time for safety monitoring and improvement. Improvement of working conditions could involve improvements to interface design, to the ergonomics of equipment, the physical working environment or the reduction of interruptions and distractions that greatly increase propensity to error. We provide one example to illustrate the potential of this kind of approach.

\section{Reducing the Burden on Staff: Simplification and Decluttering}

The improvement of the working lives of staff is a central aim of human factors work. If we want staff to spend time monitoring and improving safety we have to create time for it and not rely on enthusiasts working at weekends and in the evenings. This means that less time must be allocated to something else and decisions must be made about what can be stripped out of the current work process. We will briefly consider the issue of policies and procedures in the British NHS as an example of how we might begin to simplify the system and reduce the burden on staff.

Within the National Health Service (NHS), a vast number of policies and guidelines govern all aspects of the work of the organisation. In an analysis of clinical guidelines related to frontline care Carthey and colleagues (2011) found that in the first $24 \mathrm{~h}$ of a patient admitted for emergency surgery on a fractured neck of femur there were 76 applicable guidelines. A brief survey of 15 NHS hospitals in England who published their policies on their websites showed that they had between 133 and 495 policies covering everything from dress code to medication dispensing. The average policy was 27 pages long with length varying between 2 and 122 pages (Fig. 7.2). An average hospital has 8000 pages of policies on their websites running to more than two million words (Green et al. 2015).

The plethora of unusable quasi-legal policies is an unconscionable burden on the staff, a drain on resources and paradoxically a threat to safety. First, safety critical essential procedures and other trivial policies are not sufficiently distinguished and all formal policies become degraded. Second, staff cannot possibly comply with even a fraction of the guidelines and procedures they have to contend with. Third, huge amounts of time and resource are devoted to producing policies which are more or less unusable in practice and distract from other potentially more fruitful approaches to safety. How many such procedures can we reasonably put in place in 


\section{Guidelines for fractured neck of femur in the first $\mathbf{2 4} \mathbf{h}$}

Guidelines for investigations while patient
is In A\&E
3. Standard ECG for elderly
4. Urine dipstick
5. Blood tests
6. Chest X-ray
7. Hip X-ray
8. MRSA screen

\section{Guidelnes to monitor and manage patients} while In A\&E

9. Vital signs (full set)

10. Pain score

11. Analgesia prescribed (analgesic Ladder)

12. DVT prophylaxis

13. Oxygen administation

14. IV flulds

15. Keep nil by mouth until definitive plan made

16. Transfer to orthopaedic ward within $4 \mathrm{~h}$ of arrival

\section{Transfer to ward}

29. Transfer guidelines

30. Patient handover

\section{Pre-operative preparation guidelines}

45. Surgery within $48 \mathrm{~h}$ and during day time

46. Pre-operative assessment

47. Pre-operative fasting

48. Drug administration

49. Pre-operative nursing preparation

50. Pre-operative checks and accompanying a patient to theatres

51. Antibiotics prophylaxis prescription (intra + post-operative)

52. Consent + operation site mark

Intra-operative care guidelines

53. Theatre arrival checklist

54. Anaesthetic care (multiple)

55. Surgical safety checklist

56. Surgical operation (multiple)

57. Scrub nurse guidelines (multiple)

58. Radiation exposure

59. Sterility + laminar flow

60. Additional guidelines depending on circumstances eg bl
Post-operative care guidelines
61. Immediate care of patient in theatre recovery (multiple)
62. Post-operative infection prevention
63. Post-operative monitoring and
investigations
64. Wound care management
65. Post-operative nutrition \& supplementation
66. Post-operative surgical care, eg timing of drain removal etc.
67. Post-operative analgesia

Hospital admission

1. Procedure for triage assessment of

patient arriving in A\&E

2. Patient identification

Specific clinical guidelines

17. Does patient satisfy fast track criteria (elderly)

18. Full history and examination of every organ system

19. Assessment of social circumstances

20. Exclude \& treat other injuries

21. Patient's orientation

22. Assessment for multiple pathology

23. Assessment of injury

24. Consider possibility of elderly abuse

25. If $\mathrm{SpO}<24 \%$ check $\mathrm{ABG}$ and administer oxygen

26. Treat cardiac arrhythmias according to guidelines

27. Consider and treat community acquired pneumonia

28. Consider need for bone protection medication

\section{Ward admision/nursing checks}

31. Ward orientation, information leaflet for patient and relatives

32. Assess skin and pressure areas

33. Moving and handling guidelines

34. Nutrition assessment

35. Guidelines for clinical observations, vital signs, weight and height.

36. Continence assessment

37. Information documentation

Guidelines for managing patient care

38. Discharge planning

39. Pain management

40. Investigations

41. Drug administration

42. Patient positioning, traction, immobilization and manual handling

43. Pressure area care

44. Bone protection medication guideline for elderly care

\section{Rehabilitation guidelines}

69. Multidisciplinary assessment

for rehabilitation

70. Early post-operative mobilisation (within 24h)

71. Slips, trips and falls

72. Guidelines for exercise regime and rehabilitation

\section{Discharge planning}

73. Safe discharge and follow up

74. Involve social services if appropriate

75. Bone health assessment and treatment at discharge

Fig. 7.2 Guidelines for fractured neck of femur in the first $24 \mathrm{~h}$ 
one environment? It is ironic that so many policies and procedures are written with the aim of providing assurance and improving safety and yet the net effect is to degrade safety. They need to be drastically culled and simplified to produce a usable set of operating procedures analogous to those used in other high risk industries (Green et al. 2015).

\section{Risk Control}

Risk control strategies are used in healthcare in highly standardized and regulated environments such as pharmacy, blood products and radiotherapy where there are strict controls built into the delivery systems and restrictions on who can deliver therapies and what competencies they need. Risk control strategies could potentially be used much more widely particularly as a restraint on unnecessary or dangerous informal adaptation. Most importantly they could be used much more explicitly, with greater clarity and embraced as part of the patient safety armament. In this section we give examples of risk control strategies at both frontline and executive levels.

\section{Control of Medication}

Restrictions on the prescription and administration of drugs is a classic and widely used risk control strategy. For instance:

- There are clear guidelines about who can and cannot administer intrathecal chemotherapy (Franklin et al. 2014).

- Junior doctors are generally not permitted to prescribe certain drugs such as chemotherapy, oral methotrexate and other substances

- There are legal controls on the use of many drugs such as diamorphine and other opiates

- Nurses have to pass a test of competency to be permitted to administer intravenous medications

These restrictions are generally accepted but not thought of as a risk control strategy or as a patient safety initiative. We list them simply to make the point that risk control is already used and already accepted. The next example is rather different in being an example of the potential for risk control.

\section{Potential for 'Go and No-Go' Controls in Surgery}

Pre-flight checks require a conscious decision to proceed, referred to as a "go/ no-go" decision. The civil aviation authorities set clear criteria governing the 
acceptable conditions for flying and it is expected that aircrew will recognise situations in which risk cannot be adequately managed. In such circumstances they are empowered to cancel the flight and indeed have a clear professional responsibility to do so. In contrast in healthcare the underlying assumption is to cope and carry on even in the face of considerable risk to patients. There are comparatively few areas in which 'no go' is explicitly understood and respected in healthcare.

National guidelines on equipment standards exist in anaesthesia. If faults are detected in core equipment it must be replaced, and if a suitable replacement is not available the case should not proceed without a specific, documented reason (Hartle et al. 2012). There are parallels between aviation and the operating theatre. An operation is a complex process that depends on the correct functioning of a number of different components, both human and technical. There are certain types of equipment failures in which it is assumed no anaesthetist would proceed (for example the airway gas analyser is unavailable), a situation in which some anaesthetists would proceed (an ultrasound is unavailable for a case requiring central venous cannulation), and a situation in which most anaesthetists might be expected to proceed (hospital uninterruptible power supply is unavailable, but all primary systems are functional). In practice however, although specific guidelines exist, there are very few clear 'no go' standards and the decision is left to the theatre team who are inevitably influenced by productivity pressures and other factors (Eichhorn 2012).

'No go' conditions could be defined in surgery to protect both patients and teams by imposing an inviolable limit which can only be bypassed in cases of emergency. 'No go' conditions are objective, absolute, minimum safety standards. They correspond to the thresholds above which activities of care must stop. The no go value correspond to a stage beyond which there is no capacity for safe care whatever the other strategies.

\section{Placing Limits on Care}

As we write this section in January 2015 a number of British hospitals have declared a 'major incident'. This does not necessarily relate to any specific incident but is a statement that they have reached crisis point and are unable to cope with the volume or type of patients they are receiving. This can happen in winter when demands are high, but also at other times, for example if there is a major road accident or a large number of older patients with pneumonia. This formal declaration allows the executive team to take a number of steps:

- One of the first measures is to start postponing routine activity, such as knee and hip operations or outpatient appointments.

- Cancelling leave and calling in more staff

- Making announcements to the public that the hospital is under pressure and not to attend the emergency department unless absolutely necessary 
- In exceptional circumstances diverting ambulances so no emergency patients arrive. However, this is only used as a last resort as it increases demands on nearby sites.

This is a classic risk control strategy akin to grounding flights when an airport cannot cope with flight volume or in response to bad weather. Many hospitals take these measures in response to crisis but without necessarily having a clear cut prepared strategy in place. Risk control in its fullest sense though demands an explicit, preferably public approach to the problem to allow a considered strategic response rather than an ad hoc muddling through. Again, these critical strategies are not considered in the ambit of patient safety and are not studied, categorised, developed or taught.

\section{Monitoring, Adaptation and Response}

We have repeatedly emphasised that failures and departures from standards are not the exception but the day to day reality of healthcare. Safety is achieved partly by attempting to reduce and control such failures but also, in recognition of the impossibility of this task, by actively monitoring and managing problems that arise. The critical question is whether we leave this to ad hoc improvisation or try to build this capacity into the system (Vincent et al. 2013). Many proposed safety initiatives fall into this category but few have been implemented in a thoroughgoing and strategic manner. We provide some a small number of examples but there is huge scope for the development, formalisation, training and implementation of considered approaches to monitoring and adaptation.

\section{Patients and Families as Problem Detectors}

The active engagement and empowerment of patients and carers in an increasingly complex system poses huge challenges on many fronts. Patients and carers will have an increasingly important role in maintaining safety as home care expands, which will be discussed in the following chapter. At this point we simply want to highlight that almost all safety interventions that are aimed at patients fall into the category of monitoring, adaptation and response. In the hospital context patients and carers are in many cases being asked to compensate for problems of poor reliability and to form an additional defence against potential harm (Davis et al. 2011).

Many patient focused safety interventions are aimed at encouraging people to speak up if they notice problems with medicines, identification or other issues. More challengingly patients are asked to confront staff who have not washed their hands to support infection control (Pittet et al. 2011). Some of these interventions are entirely reasonable and in fact necessary; patients have a privileged and unique 
view of their own care and we need their insights into how safety is compromised. But we should be clear that patients are often being asked not only to check for problems that arise in complex care but to detect and compensate for problems that are not of their making.

\section{Team Training in Monitoring, Adapting and Response}

Teams, when working well, have the possibility of being safer than any one individual because a team can create additional defences against error by monitoring, double-checking and backing each other up: when one is struggling, another assists; when one makes an error, another picks it up (Vincent et al. 2010). Several authors have described how healthcare teams in emergency departments (Wears and Woods 2007) and operating theatres (Carthey et al. 2003) anticipate and thwart potential safety events. This can extend to more formal collaborative cross-checking, where one person, role, group or unit provides feedback about the viability or possible gaps in another's plans, decisions, or activities (Patterson et al. 2007). Allied to this is the development of a safety culture in which speaking openly about error is supported and indeed encouraged. Once one realises that errors and failures are inevitable, at least when the system is under pressure, the rationale for openness about error becomes clear. This kind of preparation is particularly critical in the more fluid and dynamic clinical environments where uncertainty is common and lapses frequent. For example, the WHO Surgical Safety Checklist is usually thought of as a means of checking processes such as the giving antibiotics in a timely fashion. However the checklist also prompts a brief period of reflection (the 'time out') in which members of the theatre team highlight potential problems and, by introducing each other, increases the chance of team members speaking up if problems are identified (Haynes et al. 2009; Kolbe et al. 2012).

\section{Briefings and Debriefings, Handovers and Ward Rounds}

Operational meetings, handovers, ward rounds and meetings with patients and carers are all sources of intelligence that allow the monitoring of safety For example, operational meetings held by senior managers can unblock beds and improve the flow of patients through a hospital, identify safety issues relating to infection outbreaks, and thwart the potential for unsafe discharge of patients. Briefings carried out by operating theatre teams provide an opportunity to identify and resolve equipment problems, staffing and theatre list order issues before a case starts. Debriefings carried out at the end of the theatre list support reflective learning on what went well and what could be done better tomorrow. Increasingly, briefings and debriefings are being introduced in other healthcare domains such mental health teams (Campbell et al. 2014). 


\section{Mitigation}

The treatment and remediation of physical problems is obviously necessary when a patient has suffered some harm or complication. However psychological support is equally important both for patients and staff. Organisations vary hugely in the extent to which they are willing, prepared and able to provide support emotionally, practically and financially. Some hospitals have very well established systems for responding when patients have been harmed and highly developed mitigation strategies; others simply react and adapt.

\section{Support Systems for Staff and Patients}

The basic needs of injured patients have been understood for 20 years. We would all, in varying degrees, like an apology, an explanation, to know that steps had been taken to prevent recurrence and potentially financial and practical assistance (Vincent et al. 1994). We know that staff suffer a variety of consequences from being the 'second victim' as Albert Wu eloquently expressed it, not implying that the experiences of staff were necessarily comparable to those of injured patients (Wu 2000). We should also consider that a member of staff who has been seriously affected may well be performing poorly and be a risk to future patients; this again is rarely addressed. There are a few pioneering examples of programmes of support for both patients and staff (Box 7.2) but this is an area of safety management which needs substantial development (Iedema et al. 2011).

\section{Box 7.2. Medically Induced Trauma Support Services (MITSS)}

Linda Kenney, the founder of MITSS, experienced a grand mal seizure during an operation while cared for by an anaesthetist, Frederick van Pelt. Together they founded MITSS which provides support for both patients and staff. The Peer Support Programme uses colleagues as the primary support, following an approach that has been successfully used in the police, fire and emergency medical services. The programme aims to recruit credible, experienced clinical staff with personal understanding of the impact of error who are immediately available to provide confidential reflection and support. An education and training programme runs in parallel that aims to challenge the culture of denial of emotional response to serious errors and events. The hospital concerned made an active commitment to disclosure and apology and developed an Early Support Activation (ESA) programme for patients and families. The long-term strategy is to have a comprehensive emotional support for patients, families and care providers (van Pelt 2008). 
The University of Michigan Health System pioneered a programme which included both support for patients and staff but also active intervention to provide compensation if appropriate and reduce the need for costly and potentially acrimonious litigation. The organisation performs active surveillance for medical errors, fully discloses errors to patients, and offers compensation when it is at fault. Evaluation of the programme found a decrease in new legal claims, number of lawsuits per month, time to claim resolution, and costs after implementation of the program of disclosure with offer of compensation. This approach did not increase legal claims and costs even in the notoriously litigious United States (Kachalia et al. 2010); in fact some decline in litigation was reported in Michigan generally through the latter part of the study period. Several New York hospitals have now implemented similar 'communication and resolution programmes'. To be successful they require the presence of a strong institutional champion, investment in developing and marketing the program to sceptical clinicians, and making it clear that the results of such transformative change will take time (Mello et al. 2014).

\section{Regulatory and Political Determinants of Approaches to Safety}

We have illustrated our five strategies within hospitals from the perspectives of both managers and frontline clinicians. To some degree they can determine the strategies they use to enhance safety. However they are also constrained by the wider regulatory and political environment. Regulators and politicians also have to decide on safety strategies for the wider system and their actions also determine the nature and feasibility of safety strategies within the organisations they influence. The two examples below show that the wider regulatory and political environment has a powerful influence not only on the form of healthcare that is delivered but on the safety strategies that can be adopted.

In France, the regulations governing radiotherapy, which are the province of the Nuclear Safety Agency (ASN), are much stricter than those governing the use of chemotherapy which is overseen by Haute Autorite de Sante (HAS). As a result, radiotherapists work to an ultra-safe model with many stipulations about the conditions of operation and an absolute requirement to minimise all errors and adverse events. ASN never hesitates to audit and suspend approval in cases of overdose or other serious problems. In contrast, oncologists have much greater freedom of action and are able to begin with a high dose (to bring maximum benefit) and reduce the dose as necessary depending on the patient's tolerance of unacceptable side effects. There are strict controls on the pharmaceutical production and on the preparation of chemotherapy, but comparatively few restraints on decisions about dose which are determined by the expert judgement of oncologists. These differences are in large part due to the different highlevel requirements coming from the relevant authorities. Risk controls are 

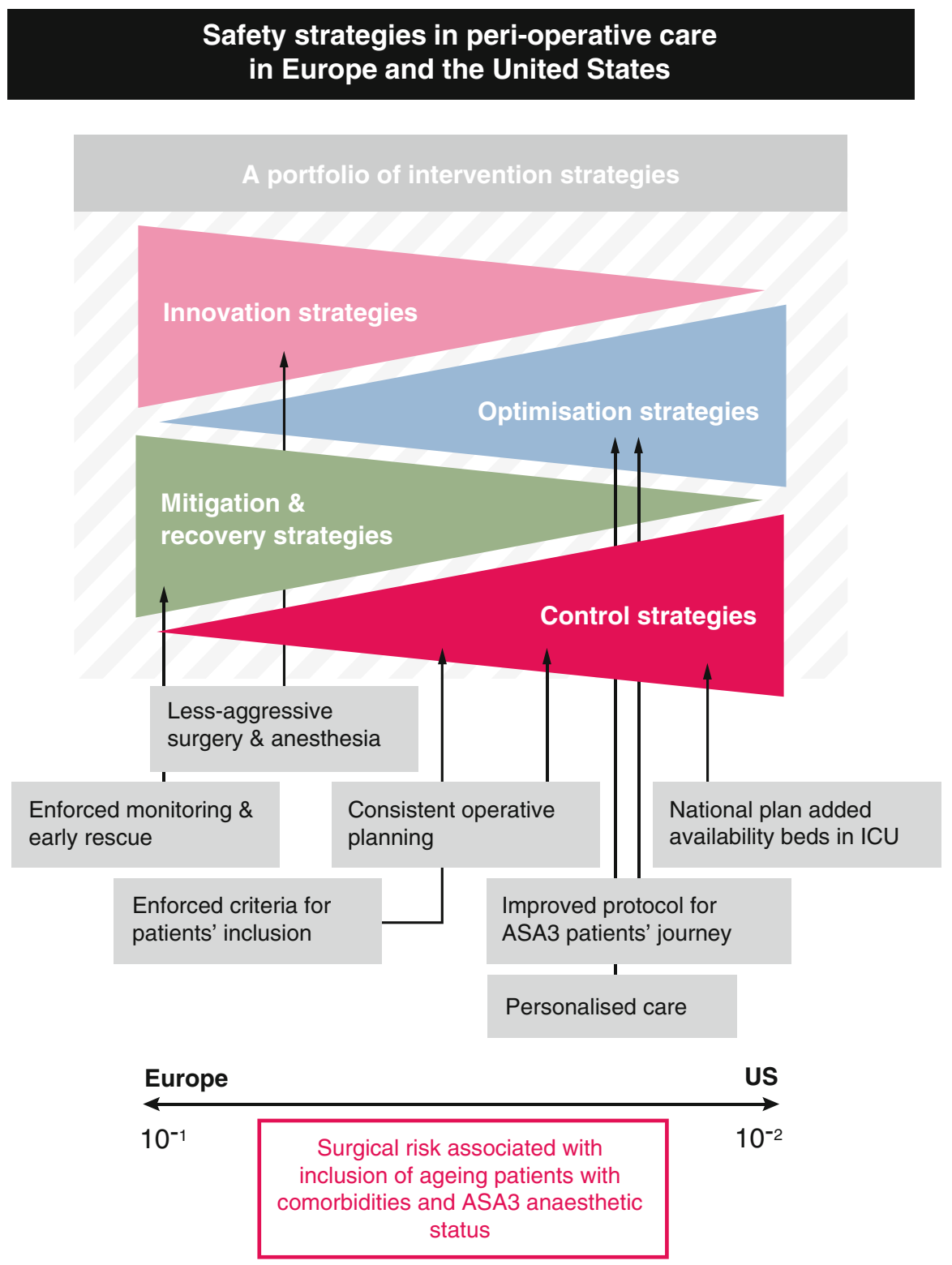

Fig. 7.3 Safety strategies in peri-operative care in Europe and the United States

imposed on radiotherapy, while much autonomy and adaptation is allowed for chemotherapy.

Different political contexts and levels of funding obviously influence the healthcare that can be delivered but also affect the safety strategies that can be employed. In this respect there are marked differences between approaches 
adopted in Europe and the United States in the surgical treatment of older patients with complex problems (Fig. 7.3). In Europe approximately $8.5 \%$ of patients having major surgery are admitted to intensive care at some point in their hospital stay; mortality can be $4 \%$ for all patients overall and as high as $20 \%$ for older patients who are a poor anaesthetic risk. In contrast in the United States, $61 \%$ of similar patients are admitted to intensive care; mortality is $2.1 \%$ for all patients and 10-15\% for older patients with anaesthetic risk. These improvements in outcome in the United States are impressive but come at a considerable cost. In 2013 , critical care services alone accounted for $4 \%$ of all US health care expenditures, or nearly $1 \%$ GDP (Neuman and Fleisher 2013). Europe has not made that choice which in turn means that different strategies must be employed which have a much stronger emphasis on the detection of problems and rapid response to mitigate the expected poorer outcomes (Fig. 7.3). In fact differences in mortality between high and low-volume hospitals are not associated with large differences in complication rates. Instead, these differences seem to be associated with the ability of a hospital to effectively rescue patients from complications. Strategies focusing on the timely recognition and management of complications once they occur may be essential to improving outcomes at low-volume hospitals (Ghaferi et al. 2009, 2011).

\section{Safety in Context: The Many Hospital Environments}

We have begun to set out the safety strategies that may be employed in hospitals, illustrating the broad strategies and the associated interventions. We recognise that much work is needed to explore this approach and map both actual and potential safety strategies and interventions. Another critical task is to consider how the strategies should be chosen and adapted to the many different environments within the hospital and in the light of the increasing complexity of care and the pressures on hospitals to provide safe care $24 \mathrm{~h}$ a day, 7 days a week.

We have previously argued that there are areas of the hospital which conform to our ultra-safe model, others which rely on a high reliability approach and number in which care is highly adaptive, albeit still with a bedrock of core procedures. In some of these settings safety is best achieved by a mixture of automation, reliable equipment and adherence to core standards and procedures. In other environments these approaches remain important but need to be complemented by a greater reliance on risk control, adaptation and mitigation. Table 7.1 provides a general illustration of how we might employ different strategies to different contexts in the hospital as we develop the right blend of interventions and modes of operation. At the moment these ideas can only be proposed. However it would be possible, in fact necessary, to begin to identify and catalogue the strategies in day-to-day use using observational and ethnographic approaches and potentially quantify the reliance on them in different contexts. 


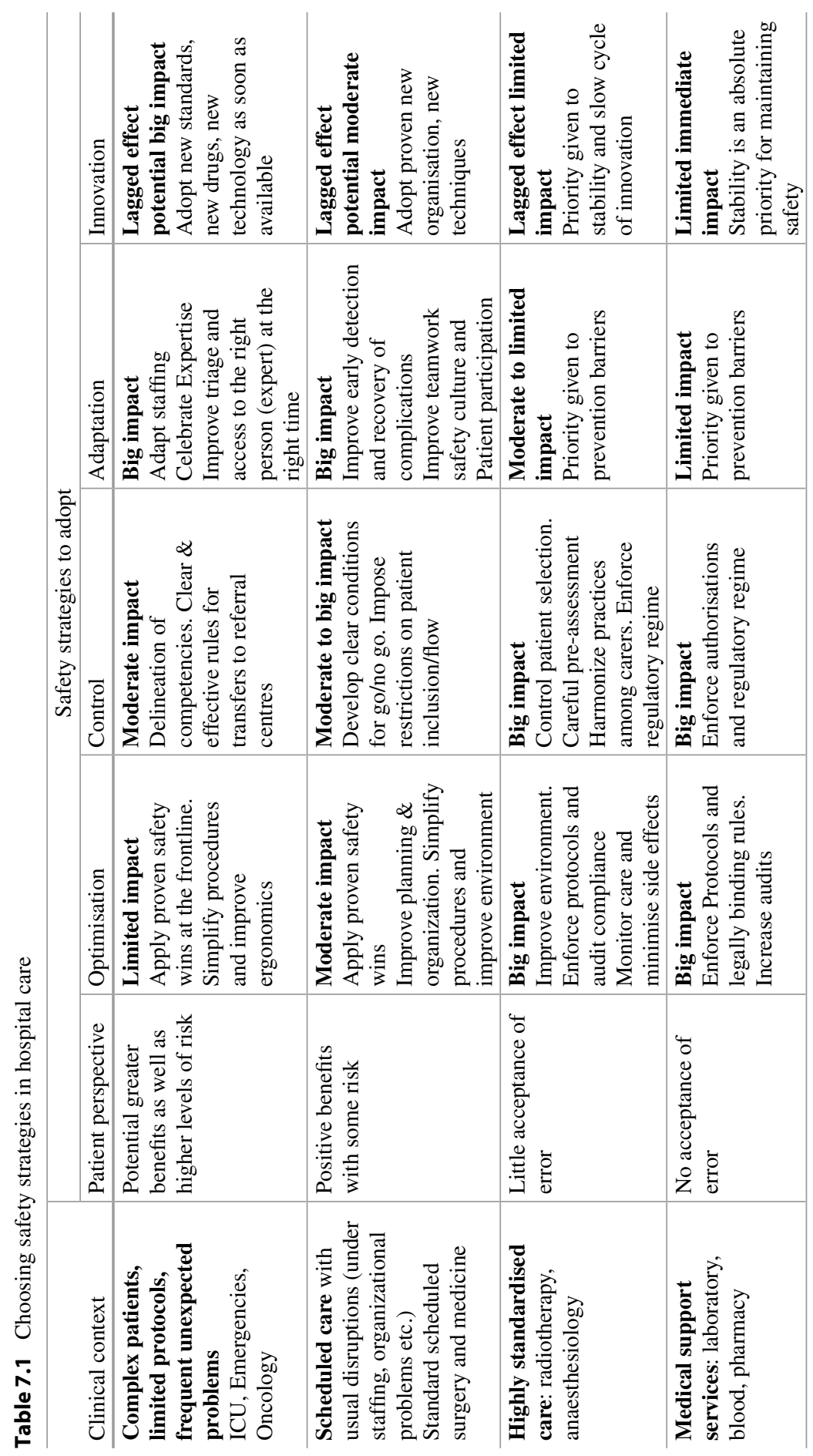




\section{Key Points}

- In the past 15 years we can distinguish three phases of patient safety each associated with different types of action and intervention: the initial establishment of clinical risk management and the drive to reduce harmful incidents; a second phase in which industrial safety concepts and methods were applied to healthcare; a third phase of focal clinical interventions, team and cultural development. The earlier strategies have continued as the new ones emerged so that we now have 'a safety layer cake'.

- Many clinical services rely very heavily on ad hoc improvisation and adaptation to compensate for deficiencies of organisation and poor reliability of basic processes. The fact that a strategy is extensively used does not necessarily mean that it is desirable

- Safety may need to be approached differently in the varying clinical contexts within the hospital. All five strategic approaches will be needed in the hospital.

- Safety as best practice. Reduction of pressure ulcers, reduction of catheterassociated infections, improved hand hygiene, improved patient identification and adherence to core standards are critical in all environments.

- Improving the system includes standardising medication formularies and protocols, the introduction of information technology in all its forms, formalising roles and responsibilities in clinical teams, the use of care bundles and daily goals to organise ward care and all efforts to improve basic working conditions.

- Risk control includes: guidelines about who can and cannot administer intrathecal chemotherapy, legally controlled drugs with restrictions on their use and the implementation of go/no go conditions for surgical operations and other procedures

- Monitoring, adaptation and recovery includes: patients and families as problem detectors, teamwork and team training to adapt and recover, the use of briefings, debriefings and handover to anticipate and respond to problems.

- Mitigation. Organisations vary hugely in the extent to which they are willing, prepared and able to provide support emotionally, practically and financially. Some hospitals have very well established systems for responding when patients have been harmed and highly developed mitigation strategies.

- The wider regulatory, economic and political environment has a strong influence on the nature of the safety strategies that are feasible to employ within the healthcare system.

Open Access This chapter is distributed under the terms of the Creative Commons Attribution Noncommercial License, which permits any noncommercial use, distribution, and reproduction in any medium, provided the original author(s) and source are credited. 


\section{References}

Association of Anaesthetists of Great Britain and Ireland (AAGBI), Hartle A, Anderson E, Bythell V, Gemmell L, Jones H, McIvor D, Pattinson A, Sim P, Walker I (2012) Checking anaesthetic equipment. Association of anaesthetists of Great Britain and Ireland. Anaesthesia 67(6):660-668

Bates DW (2000) Using information technology to reduce rates of medication errors in hospitals. BMJ 320(7237):788

Burnett S, Franklin BD, Moorthy K, Cooke MW, Vincent C (2011) How reliable are clinical systems in the UK NHS? A study of seven NHS organisations. BMJ Qual Saf. doi:10.1136/ bmjqs-2011-000442

Campbell L, Reedy G, Tritschler C, Pathan J, Wilson C, Jabur Z, Luff A, Cross S (2014) Using simulation to promote safe and therapeutic services in mental health settings. BMJ Simul Technol Enhanc Learn 1(Suppl 1):A29

Carayon P (ed) (2006) Handbook of human factors and ergonomics in health care and patient safety. CRC Press, Boca Raton

Carthey J, de Leval MR, Wright DJ, Farewell VT, Reason JT (2003) Behavioural markers of surgical excellence. Saf Sci 41(5):409-425

Carthey J, Walker S, Deelchand V, Vincent C, Griffiths WH (2011) Breaking the rules: understanding non-compliance with policies and guidelines. BMJ 343:d5283

Davis RE, Sevdalis N, Vincent CA (2011) Patient involvement in patient safety: how willing are patients to participate? BMJ Qual Saf 20(1):108-114

Eichhorn JH (2012) The Anesthesia Patient Safety Foundation at 25: a pioneering success in safety, 25th anniversary provokes reflection, anticipation. Anaesth Analg 114(4):791-800

Flin R, Burns C, Mearns K, Yule S, Robertson EM (2006) Measuring safety climate in health care. Qual Saf Health Care 15(2):109-115

Franklin BD, Panesar SS, Vincent CA, Donaldson L (2014) Identifying systems failures in the pathway to a catastrophic event: an analysis of national incident report data relating to vinca alkaloids. BMJ Qual Saf 23(9):765-772

Ghaferi AA, Birkmeyer JD, Dimick JB (2009) Variation in hospital mortality associated with inpatient surgery. N Engl J Med 361(14):1368-1375

Ghaferi AA, Birkmeyer JD, Dimick JB (2011) Hospital volume and failure to rescue with highrisk surgery. Med Care 49(12):1076-1081

Green J, Evered R, Saffer J, Vincent CA (2015) Policies: less is more. Safe healthcare requires clear and simple operating procedures. BMJ (unpublished manuscript)

Haynes AB, Weiser TG, Berry WR, Lipsitz SR, Breizat AHS, Dellinger EP, Herbosa T, Joseph S, Kibatala PL, Lapitan MCM, Merry AF, Moorthy K, Reznick RK, Taylor B, Gawande AA (2009) A surgical safety checklist to reduce morbidity and mortality in a global population. N Engl J Med 360(5):491-499

Iedema R, Allen S, Britton K, Piper D, Baker A, Grbich C, Allan A, Jones L, Tuckett A, Williams A, Manias E, Gallagher TH (2011) Patients' and family members' views on how clinicians enact and how they should enact incident disclosure: the "100 patient stories" qualitative study. BMJ 343:d4423

Kachalia A, Kaufman SR, Boothman R, Anderson S, Welch K, Saint S, Rogers MA (2010) Liability claims and costs before and after implementation of a medical error disclosure program. Ann Intern Med 153(4):213-221

Kolbe M, Burtscher MJ, Wacker J, Grande B, Nohynkova R, Manser T, Spahn DR, Grote G (2012) Speaking up is related to better team performance in simulated anesthesia inductions: an observational study. Anaesth Analg 115(5):1099-1108

Mello MM, Senecal SK, Kuznetsov Y, Cohn JS (2014) Implementing hospital-based communicationand-resolution programs: lessons learned in New York City. Health Aff 33(1):30-38

Neuman MD, Fleisher LA (2013) Evaluating outcomes and costs in perioperative care. JAMA Surg 148(10):905-906 
Patterson ES, Woods DD, Cook RI, Render ML (2007) Collaborative cross-checking to enhance resilience. Cognit Technol Work 9(3):155-162

Pittet D, Panesar SS, Wilson K, Longtin Y, Morris T, Allan V, Storr J, Cleary K, Donaldson L (2011) Involving the patient to ask about hospital hand hygiene: a National Patient Safety Agency feasibility study. J Hosp Infect 77(4):299-303

Pronovost PJ, Miller MR, Wachter RM (2006) Tracking progress in patient safety: an elusive target. JAMA 296(6):696-699

Reason J (1997) Managing the risk of organizational accidents. Ashgate, Aldershot

Reason JT, Carthey J, De Leval MR (2001) Diagnosing "vulnerable system syndrome": an essential prerequisite to effective risk management. Qual Health Care 10(Suppl 2):ii21-ii25

Sexton JB, Thomas EJ, Helmreich RL (2000) Error, stress, and teamwork in medicine and aviation: cross sectional surveys. BMJ 320(7237):745-749

Shekelle PG, Pronovost PJ, Wachter RM, Taylor SL, Dy SM, Foy R, Hempel S, McDonald KM, Ovretveit J, Rubenstein LV, Adams AS, Angood PB, Bates DW, Bickman L, Carayon P, Donaldson L, Duan N, Farley DO, Greenhalgh T, Haughom J, Lake ET, Lilford R, Lohr KN, Meyer GS, Miller MR, Neuhauser DV, Ryan G, Saint S, Shojania KG, Shortell SM, Stevens DP, Walshe K (2011) Advancing the science of patient safety. Ann Intern Med 154(10):693-696

Stanhope N, Crowley-Murphy M, Vincent C, O'Connor AM, Taylor-Adams SE (1999) An evaluation of adverse incident reporting. J Eval Clin Pract 5(1):5-12

Tsai TC, Joynt KE, Orav EJ, Gawande AA, Jha AK (2013) Variation in surgical-readmission rates and quality of hospital care. N Engl J Med 369(12):1134-1142

Van Pelt F (2008) Peer support: healthcare professionals supporting each other after adverse medical events. Qual Saf Health Care 17(4):249-252

Vincent C (ed) (1995) Clinical risk management. BMJ Publishing, London, pp 391-410

Vincent C, Phillips A, Young M (1994) Why do people sue doctors? A study of patients and relatives taking legal action. Lancet 343(8913):1609-1613

Vincent C, Taylor-Adams S, Stanhope N (1998) Framework for analysing risk and safety in clinical medicine. BMJ 316(7138):1154-1157

Vincent C, Taylor-Adams S, Chapman EJ, Hewett D, Prior S, Strange P, Tizzard A (2000) How to investigate and analyse clinical incidents: clinical risk unit and association of litigation and risk management protocol. BMJ 320(7237):777

Vincent C, Benn J, Hanna GB (2010) High reliability in health care. BMJ 340:c84

Vincent C, Burnett S, Carthey J (2013) The measurement and monitoring of safety. Health Foundation, London

Wachter RM (2010) Patient safety at ten: unmistakable progress, troubling gaps. Health Aff 29(1):165-173

Wears R, Vincent CA (2013) Relying on Resilience: Too Much of a Good Thing? In: Hollnagel E, Braithwaite J, Wears R (eds) Resilient Health Care. Ashgate, Farnham, pp 135-144

Wears RL, Woods DD (2007) Always adapting. Ann Emerg Med 50(5):517-519

Wu A (2000) Medical error: the second victim. BMJ 320:726-727 